\title{
New Hybrid System Using in Modelling Process of Hardening with Intelligent System
}

\author{
Matej Babič ${ }^{1, *}$
}

i* Jožef Stefan Institute

\begin{abstract}
Machine learning, concerns the construction and study of systems that can learn from data. Learning is the fundamental and most important element of biological intelligent systems. We use methods of intelligent system in industrial process. Moreover, with fractal geometry, we analysed the complexity of robot laser-hardened patterns. We analysed patterns hardened with different robot laser cell parameters; namely, the two parameters of speed and temperature. Fractal dimensions were calculated with a method for estimating the Hurst exponent $\mathrm{H}$ for 3D object. Also, fractal dimensions were used for pattern recognition and for describing the roughness of the hardened patterns. However, owing to the complexity of real-world problems, the development of intelligent learning systems still remains a challenging topic. For the analysis of results, we use an intelligent system method, namely, a neural network, genetic programing and regression. The general goal of Artificial Intelligence and Machine Learning research is to: develop new intelligent methods to make rational decisions based on observations; learn from experience; and automatically extract knowledge and patterns from data. Thus, this paper explores the use a hybrid method to improve existing hybrids. We present a new method for a hybrid system, based on the spiral method.
\end{abstract}

Keywords: intelligent systems, fractal dimension, Hybrid system, hardening.

\section{Introduction}

In nature there are many geometrical patterns which are irregular and cannot be described by classical Euclidian geometry. Thus we need a new method for describing the complexity and irregularity of patterns. A relatively new method is fractal geometry [1]. Recently, a concept of fractal geometry, which was developed originally for the analysis of irregular features in nature, has been finding increased applications in the fields of materials science $[2,3]$, for the characterization of microstructures. The key to fractal geometry is the fractal dimension [4 -16], which describes the complexity of a fractal and geometrically irregular microstructure. Measuring fractal dimensions has become a common practice for describing the structural properties of roughness and hardness of heat-treated materials. We use fractal geometry in mechanical engineering and in laser techniques. Laser hardening [17] is a metal surface treatment process that is complementary to the conventional aim and induction hardening processes. A high-power laser beam is used to heat a metal surface rapidly and selectively, so as to produce hardened case depths of up to $1.5 \mathrm{~mm}$, with the hardness of a martensitic microstructure, providing improved properties, such as wear resistance and increased strength. Fractal patterns are observed in the computational mechanics of elastic-plastic transitions. The origin of the term 'fractal' is the fact that some objects show a self-similarity over a wide 
length scale and possess some fractal dimension. But most of the fractal objects encountered in nature show a self-similarity that is generally random, and they are not created by deterministic rules like those for the Koch curve. Numerous objects found in nature demonstrate not exactly self-similarity, but statistical self-affinity. Statistical self-affinity implies that these objects exhibit self-similarity in some average sense, and over a certain local range of length scales. In this work, we have used a scanning electronic microscope (SEM) to discover and analyse the fractal structure of robot laser-hardened specimens. The present study is intended to use the new method of fractal geometry to describe completely the mechanical properties of robot laser-hardened specimens. Finally, the concept of fractal geometry is applied to characterize the microstructure, and derive useful relationships between the fractal dimension and microstructural features. The aim of the contribution is to outline possibilities of applying an intelligent system, artificial neural networks, genetic programming and regression for the modelling of mechanical steel properties after robot laser heat treatment, and to judge their perspective use in this field. The achieved models enable the prediction of final mechanical material properties, on the basis of decisive parameters of a laser beam influencing these properties. The modelling of the relationship was obtained by neural network, genetic programming and regression. We use new method of a Hybrid system to predict the roughness of hardened patterns.

\section{Materials preparation}

Firstly, we use tool steel standard label DIN standard 1.7225. We hardened the tool steel with a robot laser cell, polished and etched all patterns, after hardening, and used a field emission scanning electron microscope, JEOL JSM-7600F, to take microstructure pictures of hardened patterns. On these patterns we made measurements of roughness after hardening. In Fig. 1 some microstructure of a hardened pattern are presented.

In this article we present how parameter temperature and speed of the robot laser cell impact on the topography of the hardened pattern. The characterization of surface topography in important to the function of many materials. We

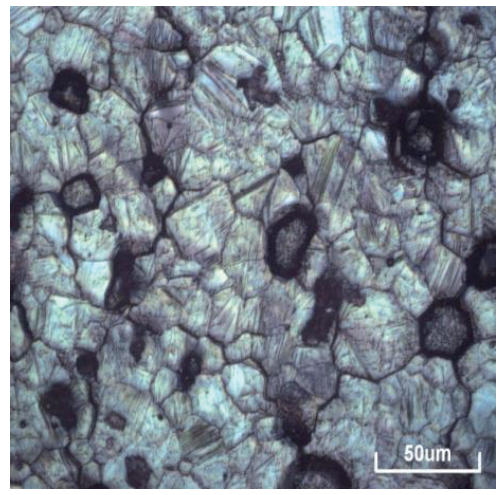

Fig. 1: Microstructure of robot laser-hardened pattern.

analyse the roughness surface of robot laserhardened patterns. Roughness is often a good predictor of the performance of a mechanical component. For the measurement of the surface roughness parameter $R_{a}$ (arithmetic mean deviation of the roughness profile) a profilometer of the robot laser-hardened patterns was used. A profilometer can measure small surface variations in vertical stylus displacement as a function of position. Fig. 2 present a roughness of robot laserhardened pattern.

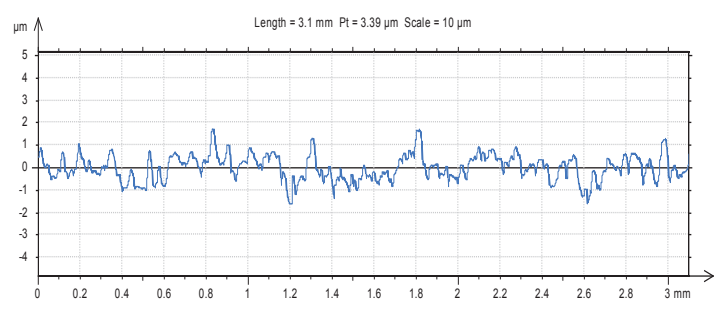

Fig. 2: Roughness of robot laser-hardened pattern.

\section{Experimental method}

The Hurst parameter [18] is understood as the correlation between random steps $X_{1}$ and $X_{2}$, which are followed by time-to-time difference $\Delta t$. The relationship between the fractal dimension $D$ and $H$ Hurst parameter is given by the equation $D=2-H$ for $2 D$ objects, and $D=3-H$ for $3 D$ objects. There is also a form called statistical selfsimilarity. With a method for estimating the Hurst exponent $H$ for $3 D$ objects [19], we analyse robot laser-hardened patterns. With fractal dimension $D=3-H$, we were able to determine the complexity of hardened patterns.

We used an intelligent system method; namely, a neural network, genetic programming and 
regression for modelling results. In information technology, a neural network (NN) is a system of programs and data structures that approximates the operation of the human brain. Researchers from many scientific disciplines are designing artificial neural networks, to solve a variety of problems in pattern recognition, prediction, optimization, associative memory, and control. An artificial neural network is composed of many artificial neurons that are linked together according to a specific network architecture. The objective of the neural network is to transform the inputs into meaningful outputs. An artificial neural network (ANN) is a machine-learning approach that models the human brain and consists of a number of artificial neurons. Neurons in ANNs tend to have fewer connections than biological neurons. Each neuron in an ANN receives a number of inputs. An activation function is applied to these inputs, which results in activation level of neuron (output value of the neuron). Conventional approaches have been proposed for solving these problems.

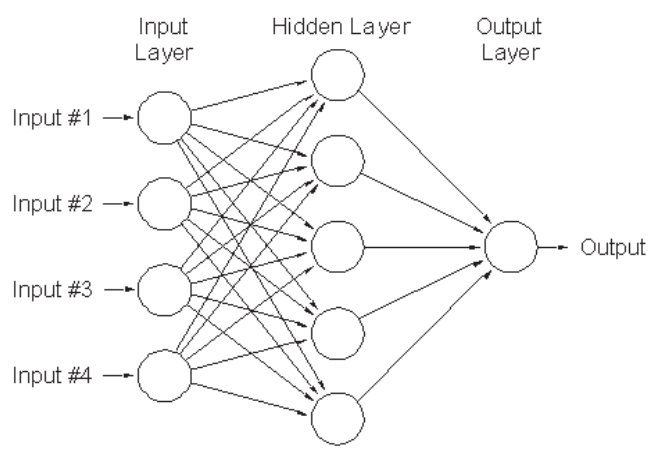

Fig. 3: Symbolic representation of the artificial neural network cell.

Genetic Programming [20] is inspired by biological evolution. It is a machine-learning technique used to optimize a solution based on a fitness score. Genetic programming may be more powerful than neural networks and other machine-learning techniques that are able to solve problems in a wider range of disciplines. The general idea behind genetic programming is: to start with a collection of functions and combine them randomly into programs; then run the programs and see which gives the best results; keep the best ones (natural selection), mutate some of the others, and test the new generation; repeat this process until a clear best program emerges. Since the dawn of the computer age, computer scientists have been trying to find a way to train computers so that they can automatically find solutions to problems; i.e. to make computers which can do something without telling them explicitly how to do it. Genetic programming (GP) is a rapidly maturing technology that is making significant strides towards applications which demonstrate that it is a sustainable science.

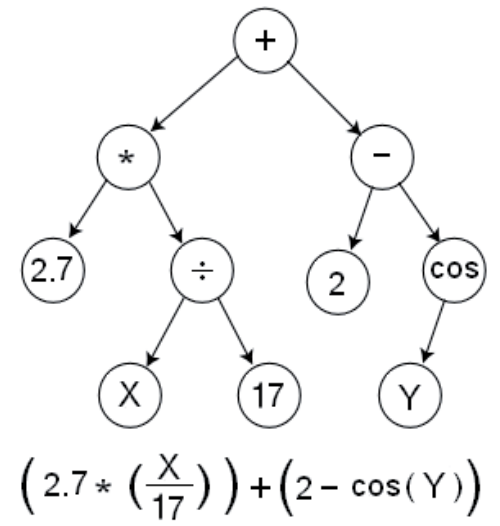

Fig. 4: Genetic programming model.

Multiple regression is a statistical tool used to derive the value of a criterion from several other independent, or predictor, variables. It is the simultaneous combination of multiple factors to assess how and to what extent they affect a certain outcome. Multiple linear regression attempts to model the relationship between two or more explanatory variables and a response variable, by fitting a linear equation to observed data. Every value of the independent variable $x$ is associated with a value of the dependent variable $y$.

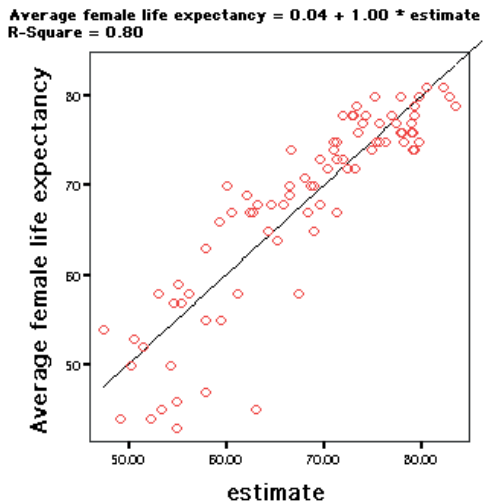

Fig. 5: Regression model. 
Hybrid evolutionary computation [21] is a robust method for solving complex global optimization problems. It can be used in practical applications in industry, especially in laser technology of hardening. As well-known method of Hybrid systems is sequences, parallel, outdoor and builtin Hybrid. In this article we present a new method of a hybrid system; spiral Hybrid (see Fig. 6). Spiral hybrid methods are connected in series in the spiral of the entrance to the method $n$. All methods work independently of the other methods. The results of input method 1 are transferred to input method $n$; the results of input method $n$ are transferred to input method 1 ; the results of input method 1 are transferred to input method $n-1$; and so on. The results of input method $i$ are transferred to the input of the spiral Hybrid. Method 1 presents Neural Network 36\% (in our case we use 8 data for a learn test set and 14 data for a test set); method 2 presents method of Neural Network 50\% (in our case we use 11 data for a learn test set and 11 data for a test set); method 3 presents method of Neural Network one live out (in our case we use 21 data for a learn test set and 1 data for a test set); method 4 presents method of multiple regression; and method 5 presents method of genetic programming.

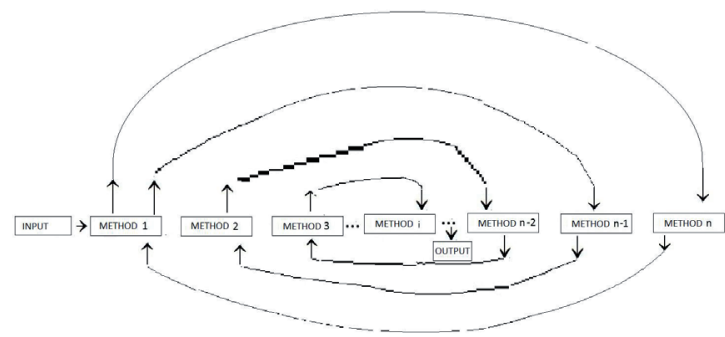

Fig. 6: Spiral Hybrid.

\section{Result and discussion}

Fig. 1 presents the microstructure before robot laser-hardening. Fig. 2 present the microstructure after robot laser-hardening with different velocity and temperature parameters. Irregular surface textures with a few breaks, represented by black islands, were revealed. Here we can see the impact of the parameters of the robot laser cell on the morphological properties of the surface. The roughness before robot laser-hardening of the material is between 24 and 1,320 nm. In all these pictures we can see the impact of the parameters of the robot laser cell on the hardness and roughness. Roughness increased after hardening by between $76 \mathrm{~nm}$ and $1.32 \mu \mathrm{m}$.

In Table 1, the parameters of hardened specimens that impact on roughness are presented. We mark specimens from $\mathrm{P} 1$ to $\mathrm{P} 22$. Parameter $\mathrm{X} 1$ presents the parameter of temperature $\left[{ }^{\circ} \mathrm{C}\right], \mathrm{X} 2$ presents the speed of hardening $[\mathrm{mm} / \mathrm{s}], X 3$ presents the fractal dimension in 3D space, and $X 4$ presents the base roughness (roughness before hardening). The last parameter is the measured surface roughness of laser-hardened robot specimens. With the fractal dimension we describe the complexity of hardened specimens. Pattern P13 has the most roughness, at 2,350 $\mathrm{nm}$. Table 2 presents experimental and prediction data regarding the surface roughness of laser-hardened robot patterns. Column 1 present patterns, column 2 presents experimental data, column 3 presents prediction with NN 36\% (in our case we use 8 data for a learn test set and 14 data for a test set), column 4 presents prediction with NN 50\% (in our case we use 11 data for a learn test set and 11 data for a test set), and column 5 presents prediction with NN one live out (in our case we use 21 data for a learn test set and 1 data for a test set). Prediction with regression is presented in column 6. Column 7 presents prediction with new method of genetic programming. Column 8 presents prediction with a new method of hybrid system. The measured and predicted surface roughness of laser-hardened robot specimens is shown in the graph in Fig. 7. The neural network model presents a $27.96 \%$ deviation from the measured data, which is less than the regression model, which presents a $133.97 \%$ deviation. The genetic programming model presents a $40.49 \%$ deviation from the measured data. The new Hybrid method presents a $55.32 \%$ deviation from the measured data.

Model Regression:

$Y=517,2268591+1,067646392 \times X 1-124,954681 \times X 2-$ $411,0529109 \times X 3+0,991431029 \times X 4$

\section{Model of regression}

$Y=71,82942368+0,006682914 \times X 1-0,172405205 \times X 2-$ $102,024491 \times X 3$

Methods of intelligent systems are very usefull. In this research we present different methods of intelligent systems to predict topographical 


$$
\begin{aligned}
& Y=X_{4}+\frac{X_{1} \cdot\left(\frac{-3,77509+X_{1}}{X_{3}}+X_{4}\right)}{\left(X_{2}+X_{3}\right) \cdot\left(\begin{array}{l}
\left(X_{2}^{2}+X_{3}\right) \cdot\left(X_{1}-\frac{3,77509}{\left.X_{1}+\frac{-3,77509+X_{1}}{X_{4}}\right)}\right. \\
\left.X_{1}+\frac{X_{4}}{X_{1}}\right)
\end{array}\right)} \\
& 3,43166 \cdot X_{1} \cdot\left(\frac{-3,77509+X_{1}}{X_{2}}+\frac{X_{1}+\frac{-3,77509+X_{1}}{X_{2}+X_{3}+X_{4}}}{X_{4}}\right) \\
& \left(X_{4}+\frac{-3,77509+X_{1}+X_{3}}{2 \cdot X_{1}^{2}}\right) \cdot\left(X_{1}-\frac{3,77509}{X_{1}}+\frac{\left(X_{2}-\frac{0,132447 \cdot\left(X_{1}-3,77509 \cdot X_{2}\right)}{X_{4}}\right) \cdot\left(X_{1}+\frac{\left(X_{2}^{2}+X_{2} \cdot X_{3}\right) \cdot\left(X_{1}-\frac{3,77509}{X_{4}}\right)}{X_{4}}\right)}{X_{4}-\frac{0,264894 \cdot\left(X_{1}-3,77509\right)}{X_{4}}}\right) \\
& +\frac{X_{1}+\frac{\left(X_{2}-\frac{0,132447 \cdot\left(-3,77509+X_{1}\right)}{X_{4}}\right) \cdot\left(X_{1}+\frac{2 \cdot X_{1} \cdot\left(X_{2}^{2}+X_{3} \cdot\left(X_{2}+X_{4}\right)\right)}{X_{4}}\right)}{X_{4}-\frac{0,264894 \cdot\left(-3,77509+X_{1}\right)}{X_{4}}}}{\left(-3,77509-\frac{3,77509}{X_{2} \cdot X_{4}}\right) \cdot X_{4}} \\
& X_{+}+\frac{\left(X_{2}-\frac{0,132447 \cdot\left(-3,77509+X_{1}\right)}{X_{4}}\right) \cdot\left(X_{1}+\frac{\left(x_{1}-\frac{3,77509}{x_{4}}\right) \cdot\left(X_{2}^{2}+X_{3} \cdot\left(X_{2}+X_{4}\right)\right)}{X_{4}}\right)}{0,26494 \cdot\left(-3,77509+X_{1}\right)} \\
& +\frac{X_{1}+\frac{0,264894 \cdot\left(-3,77509+X_{1}\right)}{\left.X_{4}-\frac{X_{4}}{X_{1} \cdot X_{4} \cdot\left(x_{2}^{2}+x_{2} \cdot x_{3}\right)}\right) \cdot X_{4}}}{\left(-3,77509-\frac{1}{x_{4}}\right.}
\end{aligned}
$$

Fig. 7: Model of genetic programming

Table 1: Parameters of hardened specimens

\begin{tabular}{|l|l|l|l|l|l|}
\hline S & X1 & X2 & \multicolumn{1}{l}{ X3 } & \multicolumn{1}{l|}{ (4 } \\
\hline P1 & 1000 & 2 & 2,304 & 24 & 201 \\
\hline P2 & 1000 & 3 & 2,264 & 24 & 171 \\
\hline P3 & 1000 & 4 & 2,258 & 24 & 109 \\
\hline P4 & 1000 & 5 & 2,341 & 24 & 76,3 \\
\hline P5 & 1400 & 2 & 2,222 & 24 & 1320 \\
\hline P6 & 1400 & 3 & 2,388 & 24 & 992 \\
\hline P7 & 1400 & 4 & 2,25 & 24 & 553 \\
\hline P8 & 1400 & 5 & 2,286 & 24 & 652 \\
\hline P9 & 1000 & 2 & 2,178 & 201 & 337 \\
\hline P10 & 1000 & 3 & 2,183 & 171 & 307 \\
\hline P11 & 1000 & 4 & 2,408 & 109 & 444 \\
\hline P12 & 1000 & 5 & 2,21 & 76 & 270 \\
\hline P13 & 1400 & 2 & 2,257 & 1320 & 2350 \\
\hline P14 & 1400 & 3 & 2,265 & 992 & 1900 \\
\hline P15 & 1400 & 4 & 2,433 & 553 & 661 \\
\hline P16 & 1400 & 5 & 2,289 & 652 & 759 \\
\hline P17 & 800 & 0 & 2,408 & 24 & 183 \\
\hline P18 & 1400 & 0 & 2,21 & 24 & 1330 \\
\hline P19 & 2000 & 0 & 2,257 & 24 & 1740 \\
\hline
\end{tabular}

\begin{tabular}{|l|l|l|l|l|l|}
\hline P20 & 950 & 0 & 2,265 & 24 & 502 \\
\hline P21 & 850 & 0 & 2,433 & 24 & 166 \\
\hline P22 & 0 & 0 & 2,289 & 24 & 24 \\
\hline
\end{tabular}

property of robot laser-hardened specimens roughness. In machine learning s Hybrid system is a very interesting method. We present $\mathrm{s}$ new method of Hybrid system: spiral Hybrid. Spiral hybrid presents a $55.32 \%$ deviation from the measured data.

\section{Conclusions}

The paper presents the use of fractal geometry to describe the mechanical properties of robot laser-hardened patterns. We use a relatively new method, fractal geometry, to describe the complexity of laser-hardened specimens. The main findings can be summarized as follows:

$\checkmark$ The results demonstrate the viable potential in using artificial neural networks, genetic programming and the regression approach in predicting roughness of robot laser hardened specimens, even with limited data sets.

$\checkmark$ We use fractal dimensions for pattern recognition. $\checkmark$ With a method for estimating the Hurst exponent $H$ for $3 D$ objects, we analyse the complexity of hardened patterns.

$\checkmark$ We found which parameters of the robot laser cell 
Table 2: Experimental and prediction data

\begin{tabular}{|l|l|l|l|l|l|l|l|}
\hline S & E & NM1 & NM2 & NM3 & R & GP & HM \\
\hline P1 & 201 & 170,21 & 174,66 & 207,30 & 411,69 & 236,76 & 130,17 \\
\hline P2 & 171 & 171,14 & 210,11 & 103,06 & 303,17 & 162,58 & 239,30 \\
\hline P3 & 109 & 104,70 & 269,68 & 171,64 & 180,69 & 113,01 & 168,77 \\
\hline P4 & 76 & 112,43 & 75,030 & 78,816 & 21,61 & 73,86 & 152,52 \\
\hline P5 & 1320 & 1333,63 & 1325,90 & 1331,7 & 872,45 & 1242,35 & 735,97 \\
\hline P6 & 992 & 948,77 & 991,560 & 967,74 & 679,26 & 984,04 & 645,40 \\
\hline P7 & 553 & 625,74 & 539,619 & 550,23 & 611,03 & 565,16 & 496,99 \\
\hline P8 & 652 & 603,49 & 485,11 & 576,24 & 471,28 & 307,72 & 500,93 \\
\hline P9 & 337 & 308,80 & 334,34 & 177,39 & 638,96 & 359,70 & 621,85 \\
\hline P10 & 307 & 209,56 & 485,97 & 484,57 & 482,21 & 288,01 & 290,34 \\
\hline P11 & 444 & 104,70 & 269,68 & 271,64 & 203,30 & 181,17 & 273,87 \\
\hline P12 & 270 & 163,41 & 65,504 & 344,28 & 127,31 & 130,63 & 235,18 \\
\hline P13 & 2350 & 1033,02 & 1303,49 & 177,00 & 2142,96 & 1774,50 & 2010,51 \\
\hline P14 & 1900 & 1085,64 & 504,01 & 1323,41 & 1689,53 & 1294,92 & 1374,78 \\
\hline P15 & 661 & 574,83 & 799,94 & 232,75 & 1060,28 & 723,30 & 1081,82 \\
\hline P16 & 759 & 414,53 & 150,69 & 802,22 & 1092,67 & 818,47 & 924,21 \\
\hline P17 & 183 & 557,65 & 503,23 & 250,85 & 477,66 & 196,09 & 432,41 \\
\hline P18 & 1330 & 1698,59 & 1038,09 & 1332,70 & 1117,02 & 533,02 & 1048,65 \\
\hline P19 & 1740 & 2058,86 & 331,29 & 1177,16 & 1746,92 & 1803,73 & 1479,58 \\
\hline P20 & 502 & 301,54 & 138,82 & 578,22 & 617,26 & 233,06 & 275,57 \\
\hline P21 & 166 & 195,58 & 137,96 & 158,90 & 495,28 & 198,64 & 163,12 \\
\hline P22 & 24 & $-53,72$ & 9,5973 & 34,118 & $-395,35$ & 23,97 & $-68,65$ \\
\hline
\end{tabular}

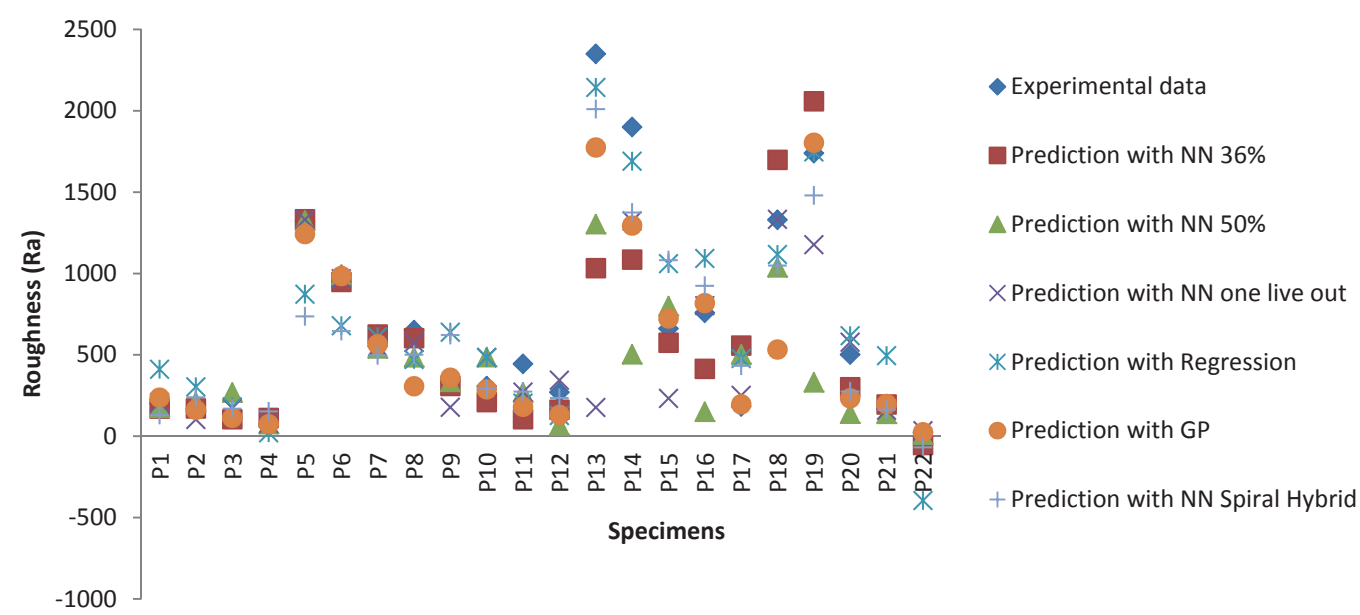

Fig. 8: The measured and predicted surface roughness of robot laser hardened specimens 
give us optimal hardness of surface.

$\checkmark$ We present a new method of Hybrid system; the spiral Hybrid.

\section{References and notes}

[1] B.B. Mandelbrot. The fractal geometry of Nature. New York: W.H. Freeman, (1982), p. 93.

[2] P.V. Yasnii, P.O. Marushchak, I.V. Konovalenko, R.T. Bishchak, Materials Science 46 (2008) 833.

[3] P.V. Yasnii, P.O. Marushchak, I.V. Konovalenko, R.T. Bishchak, Materials Science 47 (2009) 798.

[4] M. Babič, P. Kokol, M. Milfelner, S. Babič. Fractal structure of robot laser-hardened different material. V: 8th International Summer School/Conference at the University of Maribor, 26 June-10 July 2011, Maribor, Slovenia. ROMANOVSKI, Valery (ur.), ROBNIK, Marko (ur.). Let's face chaos through nonlinear dynamics: 8th International Summer School/Conference at the University of Maribor, 26 June-10 July 2011, Maribor, Slovenia. [Maribor]: CAMTP, (2011), str. 83.

[5] L.W. Fan, Y.C. Hu, T. Tian, Z.T. Yu, The predication of effective thermal conductivities perpendicular to the fibres of wood using a fractal model and an improved transient measurement technique, International Journal of Heat Mass Transfer 49 (2006) 4116-4123.

[6] T. Ficker, Fractal strength of cement gels and universal dimension of fracture surfaces, Theor. Appl. Fract. Mech. 50 (2008) 167-171.

[7] M.Q. Jiang, J.X. Meng, J.B. Gao, X.L. Wang, T. Rouxel, V. Keryvin, Z. Ling, L.H. Dai, Fractal in fracture of bulk metallic glass, Intermetallics 18 (2010) 2468-2471.

[8] A. Carpinteri, S.A. Puzzi, Engineering Fract. Mech. 73 (2006) 2110.

[9] Q. Chang, D.L. Chen, H.Q. Ru, X.Y. Yue, L. Yu, C.P. Zhang, Three-dimensional fractal analysis of fracture surfaces in titanium-iron particulate reinforced hydroxyapatite composites: relationship between fracture toughness and fractal dimension, J. Mater. Sci. 46 (2011) 6118-6123.

[10] A.L. Ahmad, N.N.N. Mustafa, Pore surface fractal analysis of palladium-alumina ceramic membrane using FrenkelHalsey-Hill (FHH) model, J. Colloid Interface Sci. 301 (2006) 575-584.

[11] B. Venkatesh, D.L. Chen, S.D. Bhole, Three-dimensional fractal analysis of fracture surfaces in a titanium alloy for biomedical applications, Scripta Mater. 59 (2008) 391-394.

[12] H.P. Tang, J.L. Zhu, Z.P. Xi, X.B. Di, J.Y. Wang, Q.B. Ao, Impact factors of fractal analysis of porous structure, Science in China Series E: Technological Sciences 53 (2010) 348-351.

[13] S.N. Kulkov, J. Tomas, S.P. Buyakova, Fractal dimension of the surface of porous ceramic materials, Technical Physics Letters 32 (2006) 73-75.
[14] S. Xie, Q. Cheng, Q. Ling, B. Li, Z. Bao, P. Fan, Fractal and multifractal analysis of carbonate pore-scale digital images of petroleum reservoirs, Mar. Petrol. Geol. 27 (2010) 476-485.

[15] H. Xie, H.W. Zhou, Z. Feng, Fractal property of spatial distribution of acoustic emissions during the failure process of bedded rock salt, Int. J. Rock Mech. Min. Sci. 48 (2011) 1344-1351.

[16] I. Dlouhý, B. Strnadel, The effect of crack propagation mechanism on the fractal dimension of fracture surfaces in steels, Eng. Fract. Mech. 75 (2008) 726-738.

[17] M. Babič, M. Milfelner, S. Stepišnik, Laser hardening metals. In: Perme, T., Švetak, D., Balič, J. (eds.), IRT Industrial Forum, Portorož, 7-8 June 2010. Source of knowledge and experience for the profession: Proceedings of the Forum. Skofljica: Profidtp.

[18] Stoev, S. Pipiras, V., and Taqqu, M. S. (2002). Estimation of the self-similarity parameter in linear fractional stable motion. Signal Processing 82, 1873-1901.

[19] BABIČ, Matej, KOKOL, Peter, GUID, Nikola, PANJAN, Peter. A new method for estimating the Hurst exponent $\mathrm{H}$ for $3 \mathrm{D}$ objects. Materiali in tehnologije, ISSN 1580-2949, (2014), 48 (2) 203-208.

[20] J. R. Koza. Course Notes for Genetic Algorithms and Genetic Programming. Spring, (2002).

[21] Vadlamani Ravi, Nekuri Naveen, Mayank Pandey. Hybrid classification and regression models via particle swarm optimization auto associative neural network based nonlinear PCA. International Journal of Hybrid Intelligent Systems. 10 (3), (2013).

\section{Biographical notes}

Matej Babič, born 7th March 1983. He received his Ph.D. degree in Computer Science from the Faculty of Electrical Engineering and Computer Science. He studied Mathematics at the Faculty of Education in Maribor. Current position: Assistant with Ph.D. in Jožef Stefan Institute. He research interests are fractal geometry, graph theory, intelligent systems, hybrid machine learning, and the topography of materials after hardening. 